How to reference this article

Scotti Jurić, R. \& Lazarić, L. (2018). La comprensione della lingua seconda. L'italiano quale materia opzionale nelle scuola croate dell'istroquarnerino. Italica Wratislaviensia, 9(1), 231-245.

DOI: http://dx.doi.org/10.15804/IW.2018.09.12

Rita Scotti Jurić \& Lorena Lazarić

Università Juraj Dobrila di Pola

\title{
LA COMPRENSIONE DELLA LINGUA SECONDA. L'ITALIANO QUALE MATERIA OPZIONALE NELLE SCUOLE CROATE DELL'ISTROQUARNERINO
}

\author{
SECOND-LANGUAGE COMPREHENSION: ITALIAN \\ AS AN OPTIONAL LANGUAGE COURSE \\ IN CROATIAN SCHOOLS IN ISTRIA AND KVARNER
}

\begin{abstract}
From a cognitive point of view, teaching to understand means teaching to activate in the most efficient way the "expectancy grammar" in order to achieve the capacity to grasp and link the logical thread that links up speech. This article evaluates level A1 of the receptive abilities in three stages of learning Italian as an optional language. The choice to analyse the same level of linguistic competence was dictated by the hypothesis that the Italian language teaching in Croatian educational tends to contain only the basic competencies. This fact could be influenced by the changes in the demographic structure of the population in the area of Istria and Kvarner, which, due to the constant flux of the population, have a negative impact on the indigenous Italian population. In the article, we will analyse a complex corpus of the comparative data (that includes 1051 participants) which resulted from the analysis of reading and listening and is based on the level of study and the sex of the interviewees.
\end{abstract}

Keywords: Italian as elective language course, Istrian and Kvarner territory, language comprehension, reading, listening 


\section{INTRODUZIONE}

T a comprensione verbale (o linguaggio ricettivo, input), a differenza della produzione (o linguaggio espressivo, output), è la capacità di decodifica di un messaggio. Si tratta di un'attività costruttiva, interattiva e attiva finalizzata a cogliere il significato del testo integrando le informazioni nuove ivi contenute con le strutture di conoscenza possedute dal lettore (cfr. De Beni \& Pazzaglia, 1995; De Beni, Cisotto \& Carretti, 2001). È un processo interattivo che considera la relazione fra parole, enunciati e turni di parola che si susseguono per costruire una coerente e coesa rappresentazione del contenuto.

La comprensione del testo è una competenza che si sviluppa precocemente già in età prescolare. Il bambino impara ad ascoltare e a estrarre da ciò che ascolta significati per lui rilevanti in un dato contesto. Le attività di ricezione e, in un secondo momento, di analisi, nell'orale come nello scritto, costituiscono una base per sviluppare competenze di produzione (delayed oral practice). Potenziare la comprensione del testo orale sembra pertanto essere fondamentale al fine di favorire lo sviluppo della comprensione del testo scritto (cfr. Palincsar \& Brown, 1984). Una buona comprensione del testo orale ha una ripercussione diretta sulla memorizzazione dei contenuti: il ricordo di un testo ben compreso è più stabile. Comprendere un testo significa quindi realizzarne una rappresentazione semantica coerente. Questo implica la capacità di trovare nessi che colleghino tra loro i concetti espressi dal testo (cfr. Zoccali, 2016).

Per quanto il processo di comprensione dei testi non sia stato ancora esplicitato e delineato con precisione, sono state avanzate delle ipotesi, alcune tese a spiegare la comprensione in termini semantico-pragmatici, altre in termini sintattici. Quello che si è riusciti a capire sin ora è che esistono tre fattori essenziali che interagiscono, favorendo o compromettendo la comprensione: l'enciclopedia, intesa come "conoscenza del mondo"; il complesso dei processi cognitivi (definizione di nessi causali, di relazioni di certezza o probabilità); la competenza comunicativa. L'interazione fra questi fattori aziona l'expectancy grammar (termine coniato da John W. Oller; 1976), per indicare la 
tendenza del parlante a prevedere cosa verrà detto in un determinato testo via via che lo recepisce. Automaticamente la comprensione non è vista come un processo lineare, parola dopo parola, frase dopo frase, ma come un processo globale e simultaneo di quanto può essere detto (cfr. Balboni, 2007). Dal punto di vista cognitivo insegnare a comprendere rappresenta una meta fondamentale e significa insegnare ad attivare nella maniera più efficace l'expectancy grammar per giungere all'intelligenza del testo, cioè alla capacità di cogliere e collegare il filo logico che lega il discorso. La strategia di ascolto o lettura può essere "estensiva" o "intensiva". La comprensione estensiva isola l'informazione che interessa e ne scarta altre, quella intensiva cerca di individuare il maggior numero di nozioni. Le strategie attivate dal processo della comprensione saranno dunque più sofisticate e articolate quando intensive, mentre saranno più schematiche quando estensive, sia nel caso che si voglia cogliere un senso globale, oppure che si voglia cogliere una specifica informazione, "comprensione mirata" (Balboni, 2007, p. 20).

\section{LE OPPORTUNITÀ DELL'ASCOLTO E DELLA LETTURA}

L'ascolto è la prima language skill che mettiamo in pratica nella vita. Implica l'identificazione di suoni, accenti, inflessioni, intonazioni ecc., e la comprensione di parole e frasi a livello orale per recepire un messaggio. Di solito, ci sono due tipi di situazioni in cui possiamo ritrovarci a utilizzare questa abilità linguistica: situazioni interattive e situazioni non-interattive. L'abilità di ascolto è uno dei modi privilegiati attraverso i quali il bambino entra in rapporto con gli altri, e va attivata e incrementata. È grazie all'ascolto di storie avvincenti fin dalla più tenera età che il bambino sviluppa l'immaginazione e la capacità narrativa e affabulatoria, nonché il piacere per la lettura. Ascoltare vuol dire prestare attenzione all'altro e costruire dei significati. Si mostra così, come detto, lo stretto legame tra le quattro abilità: ascoltare attiva processi cognitivi del tutto simili a quelli che sono richiesti in fase di lettura; entrambe queste attività sono la premessa alla produzione di alcuni tipi di scrittura, ad esempio i testi di sintesi. 
La lettura è una delle language skills che richiede formazione e pratica in quanto implica il riconoscimento dei segni grafici, la comprensione del significato delle varie parole codificate in quei caratteri e delle frasi che a loro volta queste formano. La lettura persegue tre finalità distinte: il piacere personale, l'accesso al sapere e l'inserimento nella vita sociale. La scelta di testi che rispondono ai bisogni cognitivi e affettivi dell'allievo fa sì che il piacere della lettura non si trasformi in obbligo della lettura. È fondamentale coltivare la consuetudine con i libri, rendendone facile e motivante l'accesso.

A sviluppare maggiormente queste due attività ricettive nell'apprendimento della seconda lingua è stato il comprehension approach che si riferisce a diversi metodi di apprendimento delle lingue seconde e sottolinea sommariamente la comprensione del linguaggio, più che la sua produzione (Gary \& Gary, 1981a, 1981b). Ovviamente sarà l'approccio comunicativo a pensare all'apprendimento attraverso la produzione linguistica, mettendo cioè il focus sulla parola e sulla scrittura (Lund, 1991) ${ }^{1}$. Questa prassi si fonda sul fatto che sia nell'acquisizione della lingua materna che nell'apprendimento spontaneo di una seconda lingua si registra un periodo silenzioso (Winitz, Gillespie \& Starcev, 1995). A1 contrario, l'approccio comunicativo è in gran parte incentrato sul dialogo interattivo che inizia al momento stesso dell'esposizione alla L2 (cfr. Acar, 2005). In tutti e due i casi è importante che gli studenti ascoltino un livello superiore di lingua da parte dei loro insegnanti se vogliono migliorare la loro comprensione (cfr. Thiele, 2014).

\section{METODOLOGIA DELLA RICERCA}

In questa ricerca si prenderanno in esame le abilità ricettive, quelle relative all'ascolto e alla lettura, alle quali è legato il processo di comprensione dei testi. Dall'obiettivo che ci siamo proposti sono scaturite le seguenti ipotesi: H1) la progressione dei risultati fra i tre livelli (QCER)

1 Tra gli studi linguistici sulla comprensione ricordiamo Winitz, 1981, 2003; Winitz, Gillespie \& Starcev, 1995, Krashen, 1982; Krashen \& Terrell, 1983; Asher, 1969, 1981. 
è lineare; H2) la comprensione nelle attività di ascolto è migliore che nella lettura; H3) esistono delle differenze nelle abilità ricettive tra maschi e femmine; H4) le abilità ricettive sono maggiormente facilitate nelle città bilingui.

La ricerca si limita a valutare il livello A1 del Quadro comune europeo di riferimento per le lingue: apprendimento insegnamento valutazione (Consiglio d'Europa, 2002), delle due abilità ricettive, ascolto e lettura. Il corpus è formato da alunni croatofoni che studiano l'italiano come seconda lingua, ossia come lingua opzionale, nelle scuole elementari (quinta e ottava classe) e medie superiori (terza classe) ${ }^{2}$ distribuite in nove città nel territorio istroquarnerino ${ }^{3}$. Nelle scuole croate la prima lingua straniera è l'inglese, seguita dal tedesco, e l'italiano rimane come lingua opzionale, ossia lingua che può essere scelta a piacere degli alunni. Il Test di competenza ${ }^{4}$ è stato somministrato durante le ore di italiano L2. Lo scopo è di verificare lo scarto, in positivo o negativo, rispetto ai tre livelli QCER basati sugli anni effettivi di studio dell'italiano quale L2. La scelta di somministrare lo stesso test di A1 a tutti e tre i gradi scolastici (di cui ci si attende una competenza linguistica diversificata, dettata dagli anni di studio della lingua) è stata suggerita dall'ipotesi che la lingua italiana nella verticale scolastica della scuola croata tenda ad arginarsi alle competenze di base. Il fatto potrebbe rispecchiare i cambiamenti nella struttura demografica della popolazione istroquarnerina

2 L'ordinamento scolastico croato si differenzia da quello italiano nella distribuzione della terminologia scolastica. Nel saggio verranno prediletti i termini usati in Croazia: la quinta elementare, l'ottava elementare e la terza media (superiore) che corrispondono rispettivamente, secondo il nuovo ordinamento scolastico italiano, al quinto anno della scuola primaria, terzo anno della scuola secondaria di primo grado e terzo anno della scuola secondaria di secondo grado.

3 Pola, Rovigno, Parenzo, Buie, Umago, Pinguente, Pisino, Albona e Fiume, tra cui le prime cinque sono bilingui.

4 Per le necessità della ricerca è stato stilato un questionario che comprendeva una prima parte con domande sui dati anagrafici (sesso, città) e una seconda parte, fedele all'originale Test di Certificazione di Italiano come Lingua Straniera (CILS) Livello A1 adolescenti (disponibile sul sito web del Centro CILS; si vedano Esempi prove d'esame) con tre domande per la comprensione dell'ascolto con traccia audio originale e tre per la comprensione della lettura. 
interessati da una fluttuazione costante in controtendenza rispetto alla tradizione italofona (cfr. Lazarić \& Scotti Jurić, 2017): continui esodi verso l'Italia della popolazione in età lavorativa, immigrazione nella penisola da popolazioni dell'est della Croazia, ma anche da altre parti dell'ex Jugoslavia, un dilagare della lingua inglese in tutte le sfere della società, un arresto del turismo italiano in Istria, e una mancanza generale di iniziative da parte del Governo italiano verso questi territori.

Nella ricerca sono state elaborate le risposte di 1051 alunni, 481 ragazzi $(45,8 \%)$ e 570 ragazze $(54,2 \%)$. L'età e gli anni complessivi di studio della lingua italiana quale L2 sono distribuiti come disposto nella Tabella 1.

Tabella 1: Distribuzione del corpus

\begin{tabular}{|c|c|c|c|}
\hline Classe & $V^{\text {t }}$ elementare & VIII a elementare & III ${ }^{a}$ media \\
\hline Livello $Q C R E^{*}$ & $\begin{array}{l}\text { A1 } \\
\text { livello elementare } \\
\text { contatto }\end{array}$ & $\begin{array}{c}\mathrm{A} 2 \\
\text { livello elementare sopra- } \\
\text { vvivenza }\end{array}$ & $\begin{array}{c}\text { B1 } \\
\text { livello intermedio } \\
\text { soglia }\end{array}$ \\
\hline Età & $11-12$ & $14-15$ & $17-18$ \\
\hline Anni di studio & 4 & 7 & 10 \\
\hline
\end{tabular}

* I livelli comuni di riferimento indicati sono quelli attesi, secondo la suddivisione proposta dal QCER, in base agli anni di studio di una lingua dei soggetti apprendenti.

Sono stati intervistati 364 alunni croatofoni $(34,6 \%)$ di quinta (10-11 anni), $329(31,3 \%)$ di ottava (13-14 anni) e $358(34,1 \%)$ di terza media (16-17 anni) delle Scuole elementari e medie superiori ${ }^{5}$ in lingua croata delle città di Pola $(\mathrm{N}=231,22 \%)$, Rovigno $(\mathrm{N}=119,11 \%)$, Parenzo ( $\mathrm{N}=133,13 \%)$, Buie, $(\mathrm{N}=99,9 \%)$ Pinguente $(\mathrm{N}=48,5 \%)$, Pisino $(\mathrm{N}=82,8 \%)$, Albona $(\mathrm{N}=66,6 \%)$, Fiume $(\mathrm{N}=157,15 \%)$ e Umago $(\mathrm{N}=116,11 \%)$.

L'alfa di Cronbach per la lettura risulta $\alpha=, 74$, per l'ascolto $\alpha=, 78$ e per il test complessivo $\alpha=, 83$. Il questionario è stato somministrato ai ragazzi dai loro professori di lingua italiana durante l'ora scolastica.

5 Vedi nota 2. 


\section{ESPOSIZIONE E DISCUSSIONE DEI RISULTATI}

Dai risultati ottenuti (TABELLA 2) è visibile una progressione attesa delle competenze di ascolto tra le tre classi indagate. $\mathrm{Su} 18$ risposte complessive la quinta classe ha ottenuto la media di quindici risposte esatte $(\mathrm{M}=15,60 ; \mathrm{SD}=2,629)$, l'ottava di sedici $(\mathrm{M}=16,48 ; \mathrm{SD}=2,108)$ e la terza media di diciassette $(\mathrm{M}=17,21 ; \mathrm{SD}=1,628)$.

Valori nettamente più bassi si riscontrano nella comprensione della lettura (TABELLA 2) con le seguenti medie su 12 risposte complessive: la quinta con sei risposte esatte $(\mathrm{M}=6,88 ; \mathrm{SD}=2,882)$, l'ottava con otto $(\mathrm{M}=8,55 ; \mathrm{SD}=2,834)$ e la terza media con nove $(\mathrm{M}=9,71 ; \mathrm{SD}=2,232)$. Ciò vuol dire che ci aggiriamo entro una percentuale compresa tra il 57\% e 1'81\% (GRAFICO 1).

Tabella 2: T-test per ascolto/lettura per classe (livello QCER)

Ascolto (18)

\begin{tabular}{|c|c|c|c|c|c|}
\hline Classe & media & $S D$ & $t$ & $d f$ & $p$ \\
\hline Quinta SE & 15,60 & 2,629 & $-4,889$ & 681,497 & 0,000 \\
\hline Ottava SE & 16,48 & 2,108 & & & \\
\hline Quinta SE & 15,60 & 2,629 & $-9,895$ & 607,424 & 0,000 \\
\hline Terza SS & 17,21 & 1,628 & & & \\
\hline Ottava SE & 16,48 & 2,108 & $-5,023$ & 616,083 & 0,000 \\
\hline Terza SS & 17,21 & 1,628 & & & \\
\hline \multicolumn{6}{|c|}{ Lettura (12) } \\
\hline Classe & media & & $t$ & $d f$ & $p$ \\
\hline Quinta SE & 6,88 & 2,882 & $-7,694$ & 691 & 0,000 \\
\hline Ottava SE & 8,55 & 2,834 & & & \\
\hline Quinta SE & 6,88 & 2,882 & $-14,779$ & 682,697 & 0,000 \\
\hline Terza SS & 9,71 & 2,232 & & & \\
\hline Ottava SE & 8,55 & 2,834 & $-5,920$ & 622,690 & 0,000 \\
\hline Terza SS & 9,71 & 2,232 & & & \\
\hline
\end{tabular}


Grafico 1: Percentuale risposte esatte lettura/ascolto per classe

\begin{tabular}{|c|c|c|c|c|}
\hline \multirow{2}{*}{150} & \multirow{2}{*}{$87 \%$} & \multirow{2}{*}{$92 \%$} & \multirow{2}{*}{$96 \%$} & \multirow{4}{*}{$\begin{array}{l}\longrightarrow \text { ascolto } \\
\rightarrow-\text { lettura }\end{array}$} \\
\hline & & & & \\
\hline \multirow{2}{*}{$\begin{array}{r}100 \\
50\end{array}$} & $\hookleftarrow$ & $\Rightarrow$ & $\longrightarrow$ & \\
\hline & $57 \%$ & $71 \%$ & $81 \%$ & \\
\hline 0 & quinta elementare & ottava elementare & terza media & \\
\hline
\end{tabular}

Espressa in percentuale, la comprensione dell'ascolto rientra tra 1'87\% e il $96 \%$ del profitto totale degli apprendenti (GRAFICO 1). Questo dato dimostra che i ragazzi già in quinta elementare hanno un'alta comprensione dell'italiano parlato, per cui la differenza nella comprensione dell'ascolto tra i tre livelli è più piana ed equivale al $5 \%$ tra la quinta e l'ottava e del $4 \%$ tra l'ottava e la terza media. Nelle competenze di lettura, invece, si nota uno stacco più pronunciato, pari al 14\%, tra la quinta e l'ottava elementare, e del 10\% tra l'ottava elementare e la terza media. Questo dato ci lascia perplessi, in quanto ci si aspettava una maggiore progressione dopo $3+3$ anni di studio della lingua italiana.

Tabella 3: Percentuale delle risposte esatte e t-test per le abilità ricettive complessive per classe (livello QCER)

Abilità ricettive $(30)$

\begin{tabular}{|c|c|c|c|c|c|c|}
\hline Classe & $\%$ & media & $S D$ & $t$ & $d f$ & $p$ \\
\hline Quinta SE & 74,93 & 22,48 & 4,706 & \multirow{2}{*}{$-7,483$} & \multirow{2}{*}{690,968} & \multirow{2}{*}{0,000} \\
\hline Ottava SE & 83,43 & 25,03 & 4,282 & & & \\
\hline Quinta SE & 74,93 & 22,48 & 4,706 & \multirow{2}{*}{$-14,938$} & \multirow{2}{*}{633,779} & \multirow{2}{*}{0,000} \\
\hline Terza SS & 89,73 & 26,92 & 3,139 & & & \\
\hline Ottava SE & 83,43 & 25,03 & 4,282 & \multirow{2}{*}{$-6,535$} & \multirow{2}{*}{597,917} & \multirow{2}{*}{0,000} \\
\hline Terza SS & 89,73 & 26,92 & 3,139 & & & \\
\hline
\end{tabular}

I risultati complessivi (TABELLA 3) nelle abilità ricettive (ascolto e lettura assieme) vanno dal 74,43\% nella quinta, 1'83,43\% nell'ottava fino all' $89,73 \%$ nella terza media. Pertanto, la progressione nelle abilità ricettive è rapida fino alla $\mathrm{V}^{\mathrm{a}}$ elementare e poi affievolisce nelle due fasi 
successive. I ragazzi a 11-12 anni (ossia dopo soli quattro anni di studio della lingua) raggiungono un'abilità di comprensione pari al 74,43\%, mentre negli anni successivi l'incremento è più lento: del $9 \%$ nel primo livello indagato e del 6,5\% nel secondo livello.

L'analisi t-test dimostra una differenza significativa tra le tre classi, sia separatamente per l'ascolto e la lettura sia complessivamente per le abilità ricettive (TABELLE 2 e 3). Da quanto esposto si evince che la progressione tra i livelli comuni di riferimento (A1-A2-B1) è lineare, il che conferma la prima ipotesi (H1). Le competenze degli apprendenti sono migliori nell'ascolto anziché nella lettura, per cui si conferma anche la seconda ipotesi $(\mathrm{H} 2)$.

Tabella 4: T-test per ascolto/lettura per sesso

\begin{tabular}{|c|c|c|c|c|c|}
\hline \multicolumn{6}{|c|}{ Ascolto (18) } \\
\hline Sesso & media & $S D$ & $t$ & $d f$ & $p$ \\
\hline maschio & 16,04 & 2,581 & \multirow{2}{*}{$-4,970$} & \multirow{2}{*}{866,463} & \multirow{2}{*}{0,000} \\
\hline femmina & 16,75 & 1,900 & & & \\
\hline \multicolumn{6}{|c|}{ Lettura (12) } \\
\hline Sesso & media & & $\mathrm{t}$ & $\mathrm{df}$ & $p$ \\
\hline maschio & 8,05 & 3,038 & \multirow{2}{*}{$-3,175$} & \multirow{2}{*}{982,691} & \multirow{2}{*}{0,002} \\
\hline femmina & 8,63 & 2,774 & & & \\
\hline
\end{tabular}

Legenda: livello più alto

Tabella 5: T-test per le abilità ricettive complessive per sesso

\begin{tabular}{cccccc}
\hline \multicolumn{5}{c}{ Abilità ricettive (30) } \\
\cline { 2 - 3 } Sesso & media & $S D$ & $t$ & $d f$ & $p$ \\
\hline maschio & 24,09 & 4,834 & $-4,586$ & 944,338 & 0,000 \\
\cline { 1 - 2 } femmina & $\mathbf{2 5 , 3 7}$ & 4,090 & & & \\
\hline
\end{tabular}

Legenda: livello più alto

Con l'analisi t-test (TABELLE 4 e 5) viene confermata anche la terza ipotesi (H3) secondo la quale c'è una differenza significativa nel- 
le abilità ricettive, sia viste singolarmente (ascolto e lettura) che complessivamente, tra i sessi. Di fatto, nelle medie tra maschio e femmina si riscontrano, anche se minime, una differenza di 0,71 nell'ascolto $\left(\mathrm{M}_{\text {maschio }}=16,04, \mathrm{SD}_{\text {maschio }}=2,581 ; \mathrm{M}_{\text {fermina }}=16,75, \quad \mathrm{SD}_{\text {femmina }}=1,900 ; \mathrm{t}=\right.$ $4,970 ; \mathrm{df}=866,463 ; \mathrm{p}=0,000)$, di 0,58 nella lettura $\left(\mathrm{M}_{\text {maschio }}=8,05\right.$, $\mathrm{SD}_{\text {maschio }}=3,038 ; \mathrm{M}_{\text {femmina }}=8,63, \mathrm{SD}_{\text {femmina }}=2,774 ; \mathrm{t}=-3,175 ; \mathrm{df}=982,691$; $\mathrm{p}=0,002)$ e di 1,28 nelle abilità ricettive complessive $\left(\mathrm{M}_{\text {maschio }}=24,09\right.$, $\mathrm{SD}_{\text {maschio }}=4,834 ; \mathrm{M}_{\text {femmina }}=25,37, \mathrm{SD}_{\text {femmina }}=4,090 ; \mathrm{t}=-4,586 ; \mathrm{df}=944,338$; $\mathrm{p}=0,000)$, il tutto a favore delle femmine.

Tabella 6: Analisi della varianza (ANOVA) per ascolto/lettura per città

\begin{tabular}{|c|c|c|c|c|c|}
\hline \multicolumn{6}{|c|}{ Ascolto (18) } \\
\hline Città & media & $S D$ & $F$ & $d f$ & $p$ \\
\hline Pola & $\underline{15,88}$ & $\underline{2,521}$ & \multirow{9}{*}{6,845} & \multirow{9}{*}{8} & \multirow{9}{*}{0,000} \\
\hline Rovigno & 16,35 & 2,606 & & & \\
\hline Parenzo & 17,36 & 1,208 & & & \\
\hline Buie & 16,10 & 3,069 & & & \\
\hline Pinguente & 16,92 & 1,127 & & & \\
\hline Pisino & 16,22 & 2,644 & & & \\
\hline Albona & 17,30 & 1,227 & & & \\
\hline Fiume & 16,45 & 1,607 & & & \\
\hline Umago & 16,17 & 2,300 & & & \\
\hline \multicolumn{6}{|c|}{ Lettura (12) } \\
\hline Città & media & $S D$ & $F$ & $d f$ & $p$ \\
\hline Pola & 8,39 & 2,806 & \multirow{6}{*}{24,593} & \multirow{6}{*}{8} & \multirow{6}{*}{0,000} \\
\hline Rovigno & 8,96 & 2,912 & & & \\
\hline Parenzo & 10,31 & 2,057 & & & \\
\hline Buie & 8,68 & 2,799 & & & \\
\hline Pinguente & $\underline{6,10}$ & $\underline{2.299}$ & & & \\
\hline Pisino & 7,94 & 2,941 & & & \\
\hline
\end{tabular}




\begin{tabular}{c|c|c|c|c|c}
\hline \multicolumn{6}{c}{ Lettura (12) } \\
\hline Città & media & $S D$ & $F$ & $d f$ & $p$ \\
\hline Albona & 9,79 & 1,759 & & & \\
\cline { 1 - 3 } Fiume & 7,72 & 2,594 & 24,593 & 8 & 0,000 \\
\cline { 1 - 2 } Umago & 6,51 & 3,155 & & & \\
\hline
\end{tabular}

Legenda: livello più alto; livello più basso

Tabella 7: Analisi della varianza (ANOVA) per le abilità ricettive per città

\begin{tabular}{|c|c|c|c|c|c|}
\hline & & Abilità & $(30)$ & & \\
\hline Città & media & $S D$ & $F$ & $d f$ & $p$ \\
\hline Pola & 24,28 & 4,693 & \multirow{9}{*}{15,922} & \multirow{9}{*}{8} & \multirow{9}{*}{0,000} \\
\hline Rovigno & 25,31 & 4,809 & & & \\
\hline Parenzo & 27,67 & 2,873 & & & \\
\hline Buie & 24,78 & 5,154 & & & \\
\hline Pinguente & 23,02 & 3,118 & & & \\
\hline Pisino & 24,16 & 4,862 & & & \\
\hline Albona & 27,09 & 2,410 & & & \\
\hline Fiume & 24,17 & 3,616 & & & \\
\hline Umago & $\underline{22,68}$ & $\underline{4,736}$ & & & \\
\hline
\end{tabular}

Legenda: livello più alto; livello più basso

Nell'analisi della varianza (ANOVA) viene dimostrata una differenza significativa tra le nove città indagate nell'ascolto $(\mathrm{F}=6,845 ; \mathrm{df}=8$; $\mathrm{p}=0,000)$ e nella lettura $(\mathrm{F}=24,593 ; \mathrm{df}=8 ; \mathrm{p}=0,000)$ separatamente come pure complessivamente nelle abilità ricettive $(\mathrm{F}=15,922 ; \mathrm{df}=8 ; \mathrm{p}=0,000)$ (TABelle 6 e 7).

I risultati migliori si riscontrano a Parenzo, in tutte e tre le situazioni: 17 risposte esatte su 18 nell'ascolto $(M=17,36 ; S D=2,521), 10 \mathrm{su}$ 12 nella lettura $(\mathrm{M}=10,31 ; \mathrm{SD}=2,057)$ e 27 su 30 nelle abilità ricettive 
complessivamente $(\mathrm{M}=27,67 ; \mathrm{SD}=2,873)$. Invece, per quanto riguarda i valori più bassi, i ragazzi di Pola dimostrano di avere maggiore difficoltà nella comprensione dell'ascolto $(\mathrm{M}=15,88 ; \mathrm{SD}=2,521)$, quelli di Pinguente nella comprensione della lettura $(\mathrm{M}=6,10 ; \mathrm{SD}=2,299)$ e quelli di Umago nella comprensione complessiva $(\mathrm{M}=22,68 ; \mathrm{SD}=4,736)$, ossia di ambedue le abilità.

La nostra quarta ipotesi (H4) vedeva una maggiore opportunità di comprensione nelle abilità ricettive se inseriti nelle città bilingui. In realtà questa ipotesi è stata confermata solo in parte, dato che Parenzo (città bilingue) è la città con risultati migliori sia nell'ascolto che nella lettura e, ovviamente, in ambedue le abilità analizzate congiuntamente. Il risultato di Pinguente (città non bilingue) nuovamente conferma la nostra ipotesi, visto che riporta il valore più basso nella lettura. I risultati nelle città bilingui di Pola e Umago rimangono per noi inspiegabili in quanto registrano la media più bassa, nell'ascolto la prima e nelle abilità ricettive complessive la seconda. L'unica osservazione che possiamo avanzare, ma che andrebbe verificata, è quella che l'ascolto e la lettura di per sé non garantiscono l'apprendimento. Anche se l'apprendente si trova a stretto contatto con la L2, se l'acquisizione informale non si coniuga con l'apprendimento formale non si arriverà a un livello accettabile di comprensione verbale.

\section{RIFLESSIONI CONCLUSIVE}

Implementando i due approcci contemporaneamente, la lettura e l'ascolto, meglio ancora se sono ambedue di tipo estensivo, come propongono Renandya e Jakobs (2016), si giunge ad avere un input di alta qualità e sostanzialmente più ricco, che porterà, in definitiva, a un incremento visibile dell'apprendimento della L2. La ricerca ha dimostrato che alcune considerazioni che si trascinano ancora nell'immaginario collettivo dei fruitori scolastici vanno decisamente modificate: 1'ascolto e la lettura non sono attività passive; l'ascolto e la lettura non equivalgono sempre alla comprensione (cfr. Brown, 2011). I dati ottenuti dimostrano che le due attività ricettive sono altamente collaborative e attive, e implicano una forte motivazione e applicazione da parte dell'apprendente 
per giungere a una comprensione soddisfacente. Il livello A1 del QCER analizzato in questa ricerca palesa sì una progressione linguistica, ma in minima crescita. Dopo un primo significativo profitto iniziale, l'incremento nelle classi successive è quasi nullo. Infatti, la maggioranza dei soggetti non raggiunge il livello che dovrebbe corrispondere agli anni di studio della L2 (A2 e B1). Assistiamo dunque a uno stato di stasi nello studio della lingua italiana quale materia opzionale nelle scuole croate del territorio istroquarnerino. Di conseguenza, una componente linguistica e comunicativa così bassa non incoraggia i parlanti a proseguire verso uno studio responsabile e impegnativo della lingua. Il fatto di vivere comunque in città bilingui non incoraggia lo studio della lingua italiana, intesa come lingua che si impara da sé, nel contesto della vita quotidiana. E' risaputo che la sola esposizione alla L2 non è una garanzia per giungere a una comprensione accettabile, come è il caso nelle città analizzate. Per quanto detto, l'acquisizione del materiale linguistico abbisogna di pratiche cognitive estensive e durature nel tempo, affiancate da abituali e costanti attività di lettura, affinché si possa giungere a una comprensione accettabile nella L2.

\section{BIBLIOGRAFIA}

Acar, A. (2005). The "communicative competence" controversy. Asian EFL Journal, 7(3), 55-60.

Asher, J.J. (1969). The total physical response approach to second language learning. The Modern Language Journal, 53, 3-17.

Asher, J.J. (1981). The total physical response: theory and practice. In H. Winitz (ed.), Native Language and Foreign Language Acquisition (pp. 324331). New York: New York Academy of Sciences.

Balboni, P.E. (2007). Tecniche didattiche per l'educazione linguistica. Torino: UTET.

Brown, S. (2011). Listening Myths: Applying Second Language Research to Classroom Teaching. Ann Arbor: University of Michigan Press.

De Beni, R. \& Pazzaglia, F. (1995). La comprensione del testo: modelli teorici e programmi d'intervento. Torino: UTET.

De Beni, R., Cisotto, L. \& Carretti, B. (2001). Psicologia della lettura e della scrittura. Trento: Ericson. 
Gary, J.O. \& Gary, N. (1981a). Comprehension-based language instruction: practice. In H. Winitz (ed.), Native Language and Foreign Language Acquisition (pp. 343-357). New York: New York Academy of Sciences.

Gary, J.O. \& Gary, N. (1981b). Comprehension-based language instruction: theory. In H. Winitz (ed.), Native Language and Foreign Language Acquisition (pp. 332-342). New York: New York Academy of Sciences.

Gibbons, J. (1985). The silent period: an examination. Language Learning, 35, $255-267$.

Krashen, S.D. (1982). Principles and Practice in Second Language Acquisition. Oxford: Pergamon.

Krashen, S.D. \& Terrell, T.D. (1983). The Natural Approach. New York: Pergamon.

Lazarić, L. \& Scotti Jurić, R. (2017, to appear). Le abilità ricettive degli alunni croatofoni nello studio dell'italiano L2. In R. Nikodinovska (ed.), Assessment in foreign languages \& literatures teaching. Skopje: Univerzitet "Sv. Kiil i Metodij", Filoloski fakultet "Blaze Koneski".

Lund, R.J. (1991). A Comparison of Second Language Listening and Reading Comprehension. The Modern Language Journal, 75(2), 196-204.

Oller, J.W. Jr. (1976). Evidence of a general language proficiency factor: An expectancy grammar. Die Neuren Sprachen, 76, 165-74.

Palincsar, A.S. \& Brown, A.L. (1984). Reciprocal Teaching of Comprehension-Fostering and Comprehension-Monitoring Activities. Cognition and Instruction, 1(2), 117-175.

Renandya, W.A. \& Jakobs, G.M. (2016). Extensive Reading and Listening in the L2 Classroom. In W.A. Renandya \& H.P. Widodo (eds.), English language Teaching Today: Linking Theory and Practice (vol. 5, pp. 97110). Cham: Springer International Publishing.

Thiele, J. (2014). Link Between Oral Reading And Comprehension For Second Language Learners. Davaria, Journal of Pacific Adventist University, 2, 85-92.

Winitz, H. (ed.). (1981). The Comprehension Approach to Foreign Language Instruction. Rowley: Newbury House.

Winitz, H. (2003). The Learnables, Book 1 (6th ed.). Kansas City: International Linguistics Corporation.

Winitz, H., Gillespie, B. \& Starcev, J. (1995). The development of English speech patterns of a 7-year-old Polish-speaking child. Journal of Psycholinguistic Research, 24, 117-143. 


\section{Zoccali, A. (2016). Leggere, ascoltare, comprendere. Percorsi di riabilitazione logopedica per bambini con difficoltà di comprensione del testo orale e scritto. Trento: Erickson.}

Riassunto: Dal punto di vista cognitivo insegnare a comprendere significa insegnare ad attivare nella maniera più efficace la expectancy grammar, per giungere alla capacità di cogliere e collegare il filo logico che lega il discorso. La ricerca valuta il livello $\mathrm{A} 1$ (QCE) delle abilità ricettive in tre tappe di studio dell'italiano come materia opzionale, e precisamente in quinta elementare, terza media inferiore (che nel sistema scolastico croato corrisponde all'ottava elementare) e terza media superiore. La scelta di analizzare lo stesso livello di competenza linguistica è stata dettata dall'ipotesi che la lingua italiana nella verticale scolastica della scuola croata tende ad arginarsi alle competenze di base. Il fatto potrebbe rispecchiare i cambiamenti nella struttura demografica della popolazione istroquarnerina, interessata da una fluttuazione costante in controtendenza rispetto alla tradizione italofona. Si prenderanno in esame i dati comparati di un corpus complessivo di 1051 soggetti, scaturiti dall'analisi della lettura e dell'ascolto in base al grado di studio e al sesso.

Parole chiave: italiano materia opzionale, territorio istroquarnerino, comprensione, lettura, ascolto 